\title{
Angular Projections of Fractal Sets
}

\author{
Ruth Durrer, Jean-Pierre Eckmann \\ Dépt. de Physique Théorique, Université de Genève, 24, Quai E. Ansermet, CH-1211 Genève, Switzerland \\ Francesco Sylos Labini ${ }^{1}$, Marco Montuori ${ }^{1}$, Luciano Pietronero ${ }^{1,2}$ \\ ${ }^{1}$ Dipartimento di Fisica, Universitá di Roma La Sapienza and INFM Sezione Romal, Piazzale A. Moro 2, \\ 00185 Roma, Italy \\ ${ }^{2}$ ITCP Trieste, Italy
}

\begin{abstract}
We discuss various questions which arise when one considers the central projection of three dimensional fractal sets (galaxy catalogs) onto the celestial globe. The issues are related to how fractal such projections look. First we show that the lacunarity in the projection can be arbitrarily small. Further characteristics of the projected set-in particular scaling-depend sensitively on how the apparent sizes of galaxies are taken into account. Finally, we discuss the influence of opacity of galaxies. Combining these ideas, seemingly contradictory statements about lacunarity and apparent projections can be reconciled.
\end{abstract}

The distribution of galaxies in the universe poses some intriguing puzzles. A group of physicists led by one of the authors of the present letter (L.P.), found by statistical analysis of all publically available three dimensional galaxy catalogs, that galaxies are distributed fractally with a fractal dimension of about 2 up to the largest distances available, namely $(50-100) \mathrm{h}^{-1} \mathrm{Mpc}$ [1]]. On the other hand, there are strong arguments which favor homogeneity i.e., a dimension of 3, in a range of up to $3000 \mathrm{~h}^{-1} \mathrm{Mpc}$, which is the Hubble scale [2,3]. At distances of the order of this limit, largely unknown evolutionary effects probably influence the galaxy distribution, which complicates the analysis.

In this Letter, we study some of the problems related to the observations at "large" distances up to the Hubble limit.

General theoretical arguments lead to a prediction of a homogeneous universe, which has dimension 3, while the analysis performed in [1] indicate a dimension of about 2, as far as threedimensional data are available. What is even more intriguing is that the projection of the available data onto the celestial globe shows no apparent lacunarity and this seems in flagrant contradiction with the dimension of 2 found in [1] , when one considers the data as a point set in 3 dimensions. In a widely circulated letter, Peebles has asked whether the mathematics of fractals allows for such a phenomenon.

Our-perhaps surprising-result is that if one takes finite size effects properly into account, a set of dimension 2 in 3-dimensional space can have a non-fractal and quite homogeneous projection onto the celestial globe. We will explain this result below and put it into perspective with known mathematical facts. Depending on how finite size effects are taken into account, sometimes the projection will be homogeneous and sometimes it will be fractal. In particular, we will argue that there is no contradiction between observing a fractal dimension 2 in 3-dimensional space and a uniform projection of equal size points onto the celestial globe.

Of course, this result leaves wide open the more fundamental question of how a galaxy distribution of dimension of 2 can be reconciled with the homogeneity of the universe predicted by the currently accepted cosmological models. We have no answer to this puzzle. In this letter we 
just show that absence of a unique interpretation of the measurements - to have more confidence in the data.

In order to explain the "projection paradox" and to put it into context, we first explain some mathematical aspects of the problem. A set $\mathbf{A}$ in $\mathbf{R}^{3}$ is said to have Hausdorff dimension $d$ if it can be covered by sets $S_{i, \delta}$ of diameter less than $\delta$ in such a way that the "Hausdorff measure"

$$
H^{s}(\mathbf{A})=\liminf _{\delta \rightarrow 0} \sum_{i}\left(\operatorname{diam} S_{i, \delta}\right)^{s}
$$

is zero for $s>d$ and infinite for $s<d$. For $s=d$ one can have $H^{d}(\mathbf{A})=\infty$ or $H^{d}(\mathbf{A})<\infty$. Thus, if the Hausdorff dimension is $d$, then $H^{d}(\mathbf{A})$ can be finite or infinite. When $d$ is an integer and $H^{d}(\mathbf{A})$ is finite, one can decompose $\mathbf{A}$ into a regular part (consisting of piecewise rectifiable sets of dimension $d$, such as lines or sheets) and a singular part (consisting of "dust"), see [П], Section 6.2, [5], Section 9. For non-integer $d$ the regular part is absent. Almost every projection of the regular part onto a $d$-dimensional plane is of positive measure, while all projections of the singular part (which is the interesting case for the study of galaxies) have measure 0, i.e., they are very small. Finally, if $H^{d}(\mathbf{A})$ is infinite, then a clever method, called the "Venetian blind construction" shows that the projection onto any subspace can be prescribed, and can be essentially anything we want. For example, one can construct a set in 3-space whose projection onto all twodimensional planes is a "sundial" in the sense that it shows the hour (minutes, and seconds) of the current time in Roman numerals ( [†], Section 6.3).

Thus, in the light of a strict mathematical definition, the answer to Peebles' question is that the projection is of positive measure (and hence relatively smooth) only in the cases when $d>2$ or when $d=2$ and either $H^{2}=\infty$ or $\mathbf{A}$ has a regular part. In this last case, the set $\mathbf{A}$ must contain rectifiable "lines" or "sheets." We disregard this situation as physically irrelevant because galaxies are considered as points or tiny disks in this analysis and thus form a "dust-like," i.e., irregular set.

In this Letter, we argue that this is not the whole story, because some finite size effects come into play in a subtle and beautiful way. To demonstrate this point more clearly, we first work with 1-dimensional sets in a 2-dimensional space and then illustrate the extension to 2-dimensional sets in 3-space.

We say that a set (of galaxies) has an effective dimension 1 inside a physical space of dimension 2 at length scale $r$ if the mean number $n(r) d r$ of galaxies between distance $r$ and $r+d r$ goes like $C d r$, where $C$ is a constant. Integrating from 0 to $r$ we find for the total number of galaxies, $N$ :

$$
N(r)=\int_{0}^{r} d r^{\prime} n\left(r^{\prime}\right) \sim C r
$$

and this is characteristic of the distribution of a set of dimension 1. (For a 2-dimensional set, the corresponding laws are $n(r) \sim C^{\prime} r$, and $N(r) \sim C^{\prime} r^{2} / 2$.) Eq. 2 shows the well-known fact that if the dimension of a set is not equal to the dimension of the space in which it is embedded, then the number density goes down (in our case like $1 / r$ ). This is precisely the cosmologically intriguing aspect when dealing with distributions which are not of maximal dimension.

We next study what happens when we project such a set onto the celestial globe (the unit circle in the case of a 2-dimensional universe). If the galaxies are considered just as a countable set of points, the question of the measure of their projection makes no sense, since it is equal to 0 by definition. This is, however, not what one means by the dimension of an experimentally measured set, where anyway only a finite number of points occur [6]. In fact, dimension is experimentally a 
notion which holds only over a certain range of scales. Even taking that into account, the mathematical theorems cited above tell us that the projected density has zero measure when $H^{1}$ is finite and the set is singular.

Next, we analyze in more detail various aspects which come into play when one considers the projections of galaxies onto the celestial globe. The issues we discuss here are lacunarity, the rôle of taking into account apparent sizes, and the influence of opacity. We first define these quantities. The lacunarity describes the sizes of voids between the galaxies. These voids can be large in a set of small dimension, but we shall show that they can be arbitrarily small in the projection. The apparent size problem has to do with whether we represent galaxies as points of equal size, or apparent size. The first representation will be called pixel projection and the second apparent size projection. Finally, opacity is related to the following observational problem: We assume there is a limit to how close together two galaxies can be observed, and this means that little opaque disks are drawn around each observed galaxy.

We illustrate all these phenomena for a set of dimension 1 in 2 -space. We then generalize to the case of a set of dimension 2 in 3-space. Our example is constructed as follows: Divide the unit square into 25 equal small squares and fill the central square and the four corners. We call this "Pattern 0." Similarly, we can fill 5 among 25 squares by Patterns 1, 2, and 3 as shown in Fig. 1

The fractal of Fig. 2 is then obtained by dividing the square recursively, choosing at each level randomly one of the 4 patterns. (An even more homogeneous projection is obtained by choosing at level $n$ the pattern $(n \bmod 4)$ of Fig. 1.) These fractals have dimension 1, finite Hausdorff measure $H^{1}$, and are of the singular type described above. Of course, space gets quite empty far away from the origin, but such fractals "block" the skylight in almost all directions for an observer at the center, and hence she will see an almost uniformly black sky as in Fig. \&. If the observer is not at a center, but still on a "galaxy," as we are, this argument continues to hold after enough iterations. To understand why the lacunarity decreases, we scale each point from the center to a fixed distance: $(x, y) \mapsto(x / r, y / r)$, where $r=\left(x^{2}+y^{2}\right)^{1 / 2}$. In Fig. 3, we blow each "inner" point up to a position on the outer set of squares.

Since there is always one of 4 squares colored in each of the Patterns $0-3$, we see that the apparent open space can be at most about $3 / 5^{\text {th }}$ of what it was at the previous level. Thus we get an estimate that the maximal angular void scales like $(3 / 5)^{n}$ as the number $n$ of levels grows. This answers one of Peebles' questions: There need not be any sizeable voids in the projection of a set of dimension 1 in 2-space (or for that matter, a set of dimension 2 in 3-space).

We next discuss how the projection onto the celestial globe can vary, depending on whether we show apparent size (as in Fig. 3) or just a pixel (as in the circle around Fig. (4). To simplify the discussion, we assume that all galaxies are small spheres of fixed diameter $\epsilon$. The projection of a galaxy at distance $r$ has apparent size $\sim \epsilon / r$. One can view this in one of two ways: Either the remote galaxies have very small projections (somehow less than a pixel), or the close-by ones have very large projections. Note that we discuss here for simplicity a universe of galaxies of equal size. How big is the area covered by these projections? Since

$$
H^{1}(\mathbf{A})=\lim _{\delta \rightarrow 0} \sum_{i} \operatorname{diam}\left(S_{i, \delta}\right)
$$

and there are $\sim C$ galaxies at a distance $r$ and $\operatorname{diam}\left(S_{i, \delta}\right)=\delta$, we find that the number of galaxies ( $\propto$ area of pixel projection) in a ring extending from $r_{\min }$ to $r_{\max }$ is given by

$$
N\left(r_{\min }, r_{\max }\right) \sim \int_{r_{\min }}^{r_{\max }} d r^{\prime} C \sim C\left(r_{\max }-r_{\min }\right)
$$


while the projected area is

$$
\int_{r_{\min }}^{r_{\max }} d r^{\prime} C / r^{\prime}=C \log \left(r_{\max } / r_{\min }\right)
$$

(The area is smaller when the projected galaxies start to overlap, i.e., for very fat rings.) These equations explain why projected galaxies drawn as points (Eq. Đ) look more homogeneous after increasing the size of the annulus $\left(r_{\max }, r_{\min }\right) \rightarrow \lambda\left(r_{\max }, r_{\min }\right)$; whereas a projection of the apparent area occupied by galaxies (Eq. 5) is invariant under scaling transformations. The homogenization resulting in a point projection (Eq. \) has been used as proof for the homogeneity of the universe [3]. However, in our simple fractal model, homogenization occurs as well, for shells with $r_{\max }>5^{4} r_{\min }$, and thus the reasoning in [3] is not conclusive. At small radii, finite size effects dominate, as in Fig. 1 of [3]. Using smaller sub-divisions in the construction of the example, one can lower the necessary shell-width to $r_{\max } \sim 2 r_{\min }$.

In the area projection, most of the area is covered by close-by disks, because they are larger. On the other hand, Figure 3.10 (of Radio-galaxies) in Peebles' book [2] shows all the disks of the same size (namely as small pixels, which correspond to the projected measure of a galaxy at distance about $1 / r_{\max } \sim \delta$ ).

To illustrate this point, we construct a 2-dimensional set in 3-space, using an adapted variant of the algorithm described above. (We take a $3 \times 3 \times 3$ cube and select 9 among the 27 possible little cubes, producing essentially 3 patterns with the central cube and 8 other cubes occupied. This produces higher density than $5^{3}$.) In Fig. 5 we show the apparent size and the pixel projection of this set onto the 2-dimensional celestial globe using the Aitoff-projection. One clearly notices that the pixel projection looks less "lacunary" than the apparent size projection. In Fig. 6 we show the same projection when the galaxies are "opaque." This reduces the local maxima of the apparent density of galaxies. Clearly, the projection of our 3D fractals are by far less isotropic than the observed galaxy distribution. Whether this is due to the fact that the effects discussed in this paper are not relevant to the galaxy distribution or whether it is due to our rather naive algorithm is not clear. A more detailed study of this is left as a future project. But this is not our main point here. We just want to demonstrate that from an isotropic angular projection one cannot conclude that the 3-dimensional galaxy distribution is homogeneous on any scale.

Discussion: We have analyzed voids and projected points of a 2 dimensional fractal set in 3space projected onto the celestial sphere. First, we have shown that the voids in the projection can be very small, even if they are large in the original space distribution. We also have shown that the apparent fluctuations of the projection of a set of "galaxies" depend on whether they are projected with fixed or apparent size. In the first case, the projection is more uniform than in the second. This explains in part the apparent contradiction between the relatively regular projection of a 2dimensional set in 3-space and its intrinsic fractal nature. Of course, this simple geometric analysis does not answer the much deeper apparent cosmological contradictions between the isotropy of the universe and the fractal dimension of 2 of the galaxy distribution as found in [1]]. It seems to us that this field is still wide open to speculation. Finally, if we assume that there is an observational limit to how close to each other two galaxies can be observed (by drawing a little "opaque" disks of equal size around each observed galaxy), the pixel projection such as Fig. 5 above become somewhat less fluctuating, see Fig. 6. The study of this, and similar effects in more detail will have to await further work.

Acknowledgments. We thank P. Mattila for very useful correspondence concerning fractal sets, 
and D. Pfenniger for remarks. L.P. thanks B. Mandelbrot for helpful discussions. This work was partially supported by the Fonds National Suisse.

[1] F. Sylos Labini, M. Montuori and L. Pietronero, Phys. Rep. In print (1997).

[2] P.J.E. Peebles, Principles of Physical Cosmology, Princeton University Press (1993).

[3] M. Davis, in: Critical Dialogues in Cosmology, ed. N. Turok (1997).

[4] K. Falconer, Fractal Geometry, Wiley and Sons (1990).

[5] P. Mattila, Geometry of Sets and Measures in Euclidean Spaces, Cambridge University Press (1995).

[6] J.-P. Eckmann and D. Ruelle, Rev. Mod. Phys. 57, 617-656 (1985). 

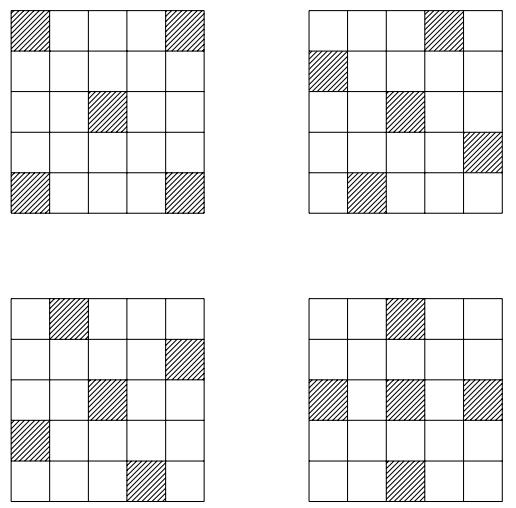

FIG. 1. The Patterns 0 to 3 used to subdivide a five by five square.
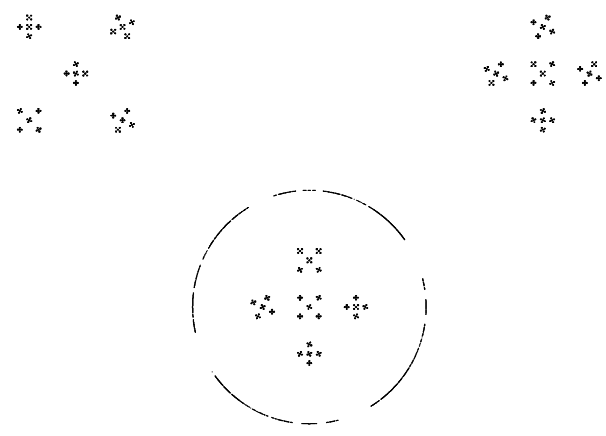

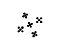

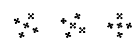

$\because \quad++^{*} x^{*} x$

$x_{x \rightarrow x}^{+x}$

FIG. 2. A fractal of dimension 1 in 2-space, and its projection onto a circle. There are 7 levels of recursion. 


\section{Fig. 3}

FIG. 3. Five levels of the same fractal as in Fig.2. Each "inner" feature is scaled outwards as shown by the lines for one feature. The corners contain the same feature as the central square. The outermost features of the central square are mapped as indicated by the radial lines. Going deeper into the recursion, each successive feature is projected clockwise to the boundary until one reaches again the corner squares in light gray which are mappings of the innermost feature.

Fig. 4

FIG. 4. The same as Fig.3, but now with "opaque" galaxies. Note that the projected density on the unit circle becomes quite uniform.

\section{Fig. 5a}

\section{Fig. $5 b$}

FIG. 5. The projection of a 2-dimensional set ( $\sim 30000$ galaxies $)$ onto the celestial globe. The calculation involves 9 levels. Top: Galaxies shown at apparent size. Bottom: Galaxies shown at equal size (pixel projection).

\section{Fig. 6}

FIG. 6. The projection of a 2-dimensional set onto the celestial globe. Galaxies are shown equal size and are opaque. 
This figure "fig3a.jpg" is available in "jpg" format from: http://arxiv.org/ps/astro-ph/9702116v2 
This figure "fig3b.jpg" is available in "jpg" format from: http://arxiv.org/ps/astro-ph/9702116v2 
This figure "newfig3.jpg" is available in "jpg" format from: http://arxiv.org/ps/astro-ph/9702116v2 
This figure "fig4.jpg" is available in "jpg" format from: http://arxiv.org/ps/astro-ph/9702116v2 
This figure "newfig4.jpg" is available in "jpg" format from: http://arxiv.org/ps/astro-ph/9702116v2 